\title{
High Speed Data Acquisition Systems
}

\author{
Richard D. Talmadge \\ Senior Electronics Engineer \\ AF Flight Dynamics Laboratory \\ Aeronautical Systems Division \\ Wright-Patterson AFB, Ohio
}

\author{
Mansour Radmand \\ Engineering Manager \\ Aydin Vector Division \\ Newtown,PA
}

\begin{abstract}
Air Force systems testing today requires that more and more data be acquired to a higher degree of accuracy and in fewer flights. This necessitates a new approach to dynamic data acquisition system design. In the past data acquisition organizations used either direct or FM recording techniques of one sort or another to acquire data for post test processing.

This paper will outline the direction that this organization is taking to reduce the size of the installed system as well as the time and money required to maintain the system during the testing process. The system discussed provides a capability to acquire both static (DC) data and dynamic data up to 10,000 Hertz and has a dynamic range in excess of $120 \mathrm{~dB}$.
\end{abstract}

\section{BACKGROUND}

The Flight Dynamics Laboratory (FDL) acquires vast quantities of data during both flight and laboratory (or ground) tests. These data have been routinely recorded on magnetic tape primarily using FM techniques. Test annotation such as time code and voice are traditionally recorded using direct record electronics. The purpose for using direct record for time is to allow the time to be read during high speed tape operations (i.e. tape search).

Since about 1967 FDL has been using Automatic Gain Ranging Amplifiers (AGRAs) as signal conditioning for all of the transducers commonly used. These early AGRAs were built on a 4x5 inch card and used discrete parts. The performance of these amplifiers was reasonably good and for the first time no ä priori knowledge of the transducers output was required for a given test condition in order to establish the appropriate amplifier gain setting.

In 1982 FDL began a program with Aydin Vector to design and develop a hybrid form of the AGRA (Ref. 1). The design goals were to provide a module that would meet the MilSpec temperature range and significantly improve the performance of the previous device. 
This program was completed in 1985 and delivered a device that has a total error band of approximately $+/-0.5 \mathrm{~dB}$ over a temperature range of $-25 \mathrm{C}$ to $85 \mathrm{C}$. The contract was modified in 1983 to include a Dual Tracking Constant Current Source (Fig. 1) for powering strain gage bridges or bridge type transducers. This device provides two current sources that track within $0.003 \%$ over the entire operating range (Ref. 2 ).

\section{$\underline{\text { APPROACH }}$}

With these tools available FDL began to look at the future in data acquisition. We assembled a system consisting of the 1967 vintage AGRAs and a Base10 Pulse Code Modulation (PCM) system. A digital multiplexer was fabricated to handle the gain codes from the AGRAs. This 120 channel system (Ref. 3) with anti-aliasing filters was constructed for and used to acquire modal data from an F-16 Ground Vibration Test (GVT). This proved to be extremely successful and this system or parts of it are still in use today.

With the experience gained from the digital data acquisition system a contract was let to Aydin Vector (the developer of the hybrid AGRA) to design and build a 16 channel multiplexer using the new AGRA as the signal conditioner. The multiplexer would provide simultaneous sampling of all the channels and produce a PCM output stream. A few of the design goals of this system were: (1) programmability (2) 20,000 Hertz per channel bandwidth, (3) 12 bit A/D, (4) parallel output for future expansion, (5) computer control and (6) the ability to synchronize multiple units in order to provide simultaneous sampling across the system. A decision was made during the design phase to reduce the last filter in the AGRA to $10 \mathrm{~K}$ Hertz in order to allow 8 channels of data to be acquired at $4.25 \mathrm{mbps}$. Three units were delivered in March of 1987 in time to support the A-10 Gun Bay Test at McClellan AFB in Sacramento, CA (Fig. 2).

In addition to these full scale multiplexers a special purpose unit was built and delivered in June of 1986.

\section{THE SYSTEM}

The first system to be delivered was a twelve channel unit that was designed to acquire data during a captive carry missile test. This system was designed to fit in a very confined space and therefore, had to be limited in scope. The multiplexer has a per channel bandwidth of 500 Hertz and originally was automatic gain range only. Since delivery, the unit has been modified to provide for programmable gain. This multiplexer functioned as expected and provided valuable performance data for this technique. 
Several of the tests that have been conducted using the 12 channel multiplexer required telemetering the data to an FDL van for near-real-time processing. The data integrity is directly related to the TM signal quality. The worst case errors occur when dropouts cause data frames to be lost. This creates an FMing effect resulting in the spectral magnitude being reduced while the bandwidth of the resonances are increased (an apparent increase in system damping). The dynamic data case is very different from the quasi static data case where simple "wild point" editing can be employed. Care must be taken to keep these effects to the absolute minimum. The same effect can be observed when using a tape recorder if the calibration and setup are not accomplished with the utmost regard for this problem.

The Programmable Dual Current Source (PDCS) module (Fig. 1) provides a means to simplify the calibration and conditioning of strain gage bridges and bride-like transducers.

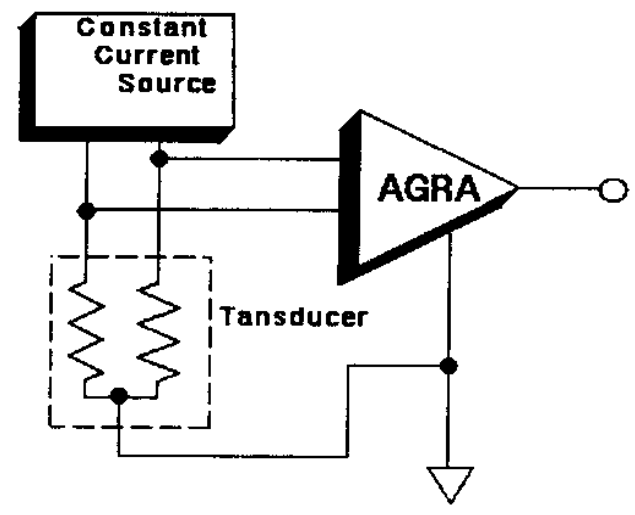

FIGURE 1. CONSTANT CURRENT EXCITATION

The use of constant current technology to condition strain gages provides many benefits. First, the line impedance is reduced which increases the noise immunity and second calibration is reduced to a simple calculation as follows:

$$
\mathrm{e}=\mathrm{I} \text { R K }
$$

where

$$
\begin{aligned}
& \mathrm{e}=\text { the output voltage } \\
& \mathrm{I}=\text { the applied current } \\
& \mathrm{R}=\text { the gage resistance } \\
& \text { and } \quad \mathrm{K}=\text { the gage factor }
\end{aligned}
$$

If the dual tracking current source is used for a conventional strain gage bridge the circuit must be modified (refer to Fig. 1). The gages in tension are serially stacked in one leg while the gages in compression are similarly placed in the opposite leg. Since this circuit is 
perfectly balanced (or symmetrical), lead wire effects and circuit noise are cancelled at the amplifier and only the desired (i.e. the signal proportional to the physical phenomenon) output remains. Even the indicated strain due to temperature change is removed. The gage sensitivity due to changes in temperature is not corrected. The PDCS module (which has programmable output from 0.25 to 16 milliamps) is used in front of a multiplexer channel for pre-conditioning strain gage bridges. The multiplexer also has a single fixed constant current source for powering transducers with internal electronics. This current supply may also be used to power single element strain gages for dynamic measurements.

The fully programmable 16 channel multiplexer has been used on three tests to date. It provides the user with a data acquisition system that has significantly improved performance. The multiplexer performance features are listed here:

1. Selectable data bandwidth(6 pole Butterworth) - 500,2000,5000,10000 Hertz

2. Gain Ranging modes - a. Automatic gain changing

b. Down range only

c. Manually programmable

3. Gain ranges of $0.25,1,4,16,64,256,1024$

4. DC accuracy - +/- $0.1 \%$

5. Max. error band $-+/-0.5 \mathrm{~dB}$

6. Full scale $-+/-10$ volts (7 volts RMS)

7. Noise (RTO) < 3 mv RMS @ 1024

8. PCM output rates $-26.5 \mathrm{kbps}$ to $4.25 \mathrm{mbps}$

9. Channel selectability $-1,2,4,8$ and 16

10. Frame length $-64-16$ bit words +16 bit frame sync

11. Frame sync pattern - Barker code (1110111110010000) 


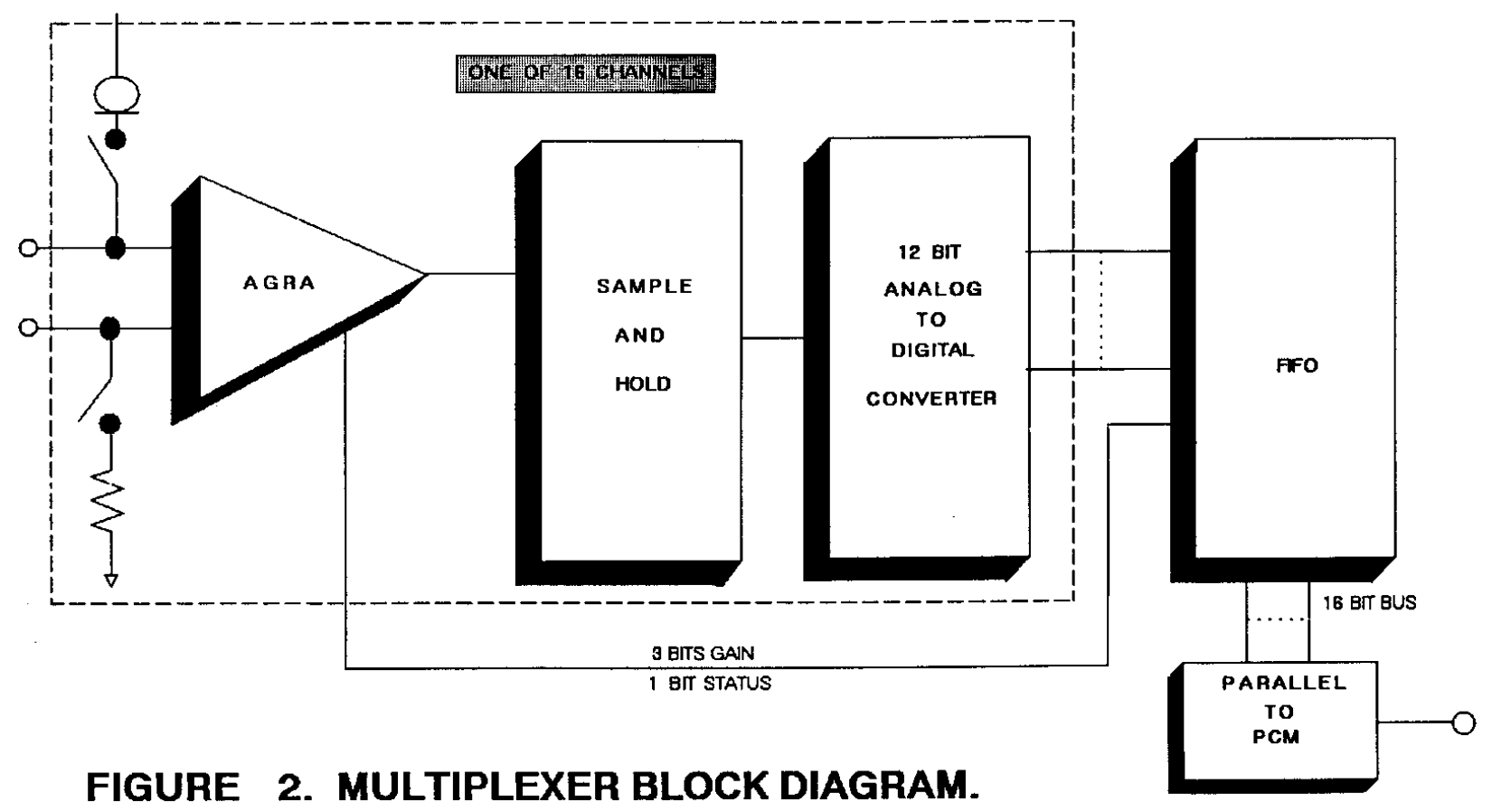

The current design is limited to 4.25 megabits per second since there are few if any recorders that can be used above this rate. Randomized NRZ-L is the code of choice; however, there is a secondary NRZ-L output. The system has the capability of being synchronized in a master slave mode so as to allow all system channels to be simultaneously sampled. Each multiplexer in the system produces its own output stream.

The major frame of the PCM stream appears as a super commutated structure. There are $\mathrm{n}$ repeats of each channel in the frame (depending on the number of channels in the scan). For 16 channels $n$ would be 4 for 64 words between frame sync patterns. For 8 channels $n$ would be 8 and so on. The scanning interleaves the channels in a $1,2,3, . . n, 1,2,3, \ldots . n$ sequence.

To minimize the overhead, a sixteen bit Barker code is inserted once every 64 words. This increases the bit rate by only $1.5 \%$. Sample periods are kept equal by buffering the data from the A/D to the output, such that sync information can be inserted without any interruption of the data sampling. The buffers are reset at the end of each frame cycle to maintain the timing relationship between each channel within the multiplexer, and between the multiplexers in the master/slave configuration.

Master/slave connection is achieved by connecting the clock and frame sync from one multiplexer to another. In this configuration the time correlation of any two channels in the system is guaranteed to be less than 25 nanoseconds excluding the propagation delay in the clock lines between the multiplexers. 
During the design phase of the contract with Aydin Vector, size was a consideration; however, performance was more important. The delivered multiplexer is large at 36 cubic inches per channel but steps are underway to cut the size in half.

\section{APPLICATION}

The first test conducted with the 16 channel multiplexers required the acquisition of $962 \mathrm{k}$ Hertz dynamic measurands and twenty static pressures and temperatures. Only three multiplexers were available for the test. (Had more been available there was no room for them in the airplane.)

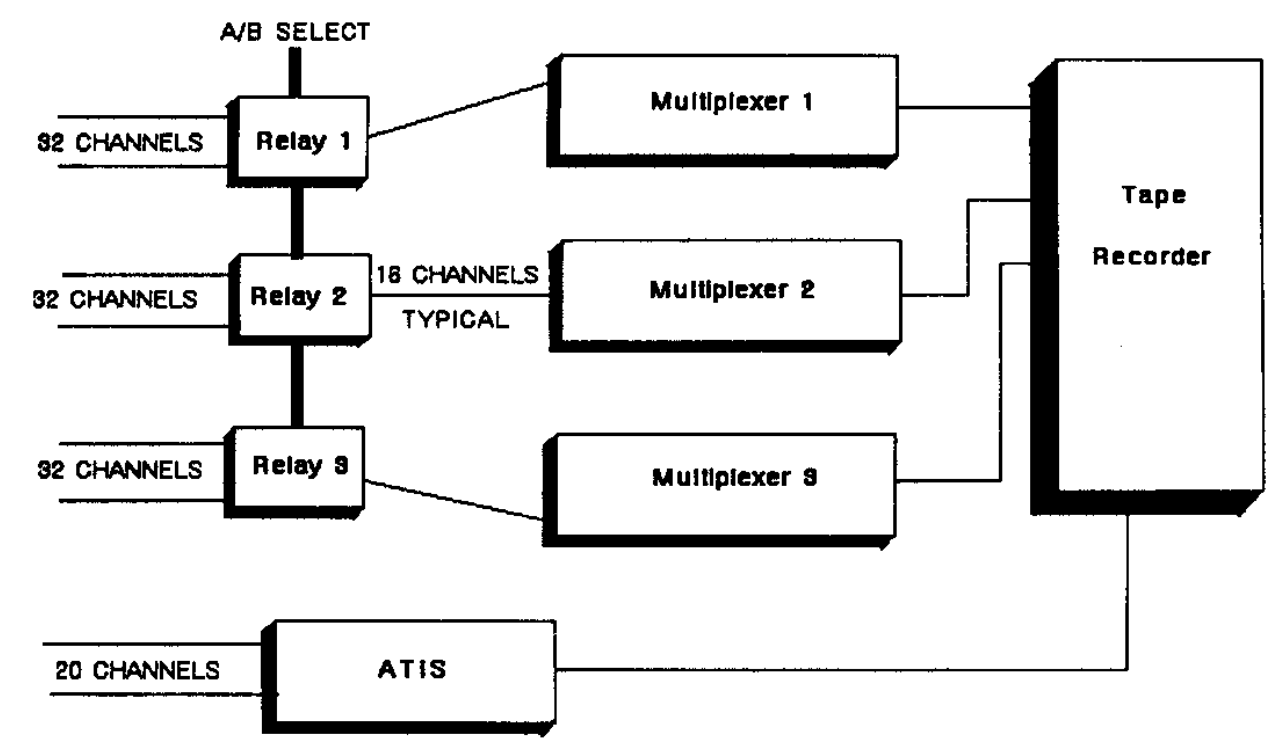

\section{FIGURE 3. A-10 SYSTEM BLOCK DIAGRAM.}

Therefore, it was decided to use an alternate approach to handle the static measurements and dynamics separately. FDL devised a method of group selection of the dynamic transducers and used another PCM conditioner/encoder for the static data. Three "T-Bar" 36 pole double throw relays were added (one in front of each multiplexer) to provide a group A and B transducer selection (see Fig. 3). With this configuration a record with the selector in the A position was taken and then group B was selected and the event repeated. This approach allowed for the acquisition of all 96 channels of dynamic data in one flight.

As discussed previously the problems associated with PCM recording must be addressed. Dropouts in the data must be held to an absolute minimum. Bit errors can be handled; however, they greatly increase the processing time. To reduce these effects; which are primarily due to the recorder characteristics, FDL modified the setup procedure for the tape recorder that was used on this test. The primary problem was traced to mechanical vibration in the tape/head interface. The tape tends to change tension across the head when 
the transport is subjected to vibration. The changing tension causes a variation in saturation of the tape which in turn results in a fluctuation (amplitude modulation) in the output on playback. Also, it was noted that when the setup procedure in the manual was followed the output level (from channel to channel) varied as much as three-to-one. It was not confirmed as to whether this was due to the condition of the heads in our machine or whether this is a normal occurrence; however, it is indicative of magnetic flux densities changing from head to head. In any event a modified procedure was developed to correct the problem. Since second harmonic distortion (time base errors or zero crossing displacement) is the primary concern for RNRZ-L recording it was decided to let the third harmonic distortion vary in order to maintain the output level constant from channel to channel and to increase the over-bias from the specified $1 \mathrm{~dB}$ to $3 \mathrm{~dB}$. This increase in bias level was to boost the saturation of the tape to help compensate for the vibration. This solved the dropout problem and produced tapes that were for all intents and purposes dropout free.

\section{FUTURE}

Since most of the tests performed require more capability then just wideband dynamic data acquisition, plans to incorporate a digital interface have been formulated. This interface will provide a method of including a secondary PCM stream as a sub frame in the multiplexer. By replacing one of the wideband signal conditioner cards with this card a multiplexer such as a Base10 Programmable data acquisition system (Pdas) can be operated as a synchronous sub frame. The interface card will provide the clocks for the sub-system and will receive word format data. This will allow for the convenient addition of such parameters as static pressures, temperatures, embedded time code, 1553 data, etc. In a typical test where $2 \mathrm{~K}$ Hertz data is being acquired, if one channel was replaced with this card a base 10 system could sample 100 channels of additional data approximately 65 time per second.

The tape recorder issue still needs to be addressed. There are future requirements that simply can not be met with the current technologies. These future requirements fall into two categories: (1) 5 to 15 megabit rate recorders for testing missiles and small fighter aircraft and (2) the larger aircraft and ground (field) testing where bit rates of up to 160 megabits per second are required. The first recorder needs to have a record time in excess

of one hour (preferably two), while the second can be somewhat less because, generally, the transport is accessible for tape changes. Both transports must meet rigorous vibration and environmental test standards. 


\section{CONCLUSIONS}

With the rising cost of flight testing today methods of reducing the overall cost, while increasing the quantity and quality of data acquired, is mandatory. The use of Automatic Gain Ranging Amplifiers (AGRAs) is an approach to solving one of the most critical issues and that is the loss of data due to the inability of the flight test engineer (for what ever reason) to determine, prior to flight, the exact output range of his transducers. This is further complicated by the restricted dynamic range of current systems. The AGRA addresses this area with a dynamic range of $66 \mathrm{~dB}$ in one gain step and an overall range of greater than $120 \mathrm{~dB}$.

The use of multiplexers such as the one outlined in this paper take a giant step towards reducing the present and future costs of flight testing. At todays cost of nearly $\$ 60,000.00$ per hour if one flight is saved the capital investment of the system can be written off.

\section{REFERENCES}

1. Talmadge, $\mathrm{R}$ and Liron, E, "Automatic Gain Ranging Amplifier", International Telemetering Conference Proceedings Vol XIX, 1983.

2. Talmadge, R, "Non-Self Generating Transducer Signal Conditioning Technique", Twelfth Transducer Workshop, Melbourne, F1 1983.

3. Talmadge, R and Banaszak, D., "Digital Data Acquisition System for Modal Testing", Sound and Vibration Magazine, November 1985

4. Strock, 0. J., "Telemetry Computer Systems", Instrument Society of America, P.O. Box 12277, Research Triangle Park, NC 